\title{
Contemporary wind generators
}

\author{
Udochukwu Bola Akuru \\ Maarten J Kamper \\ Department of Electrical and Electronic Engineering, Stellenbosch University, Stellenbosch, South Africa \\ Paper presented at the Domestic Use of Energy Conference, 19 - 20 August, 2014, Cape Town, South Africa, \\ and published in the conference proceedings. Reproduced with permission. Not peer-reviewed for JESA.
}

\begin{abstract}
It is believed that wind energy is growing at a very rapid rate, especially in the last few years. When compared with other sources of renewable energy in the energy portfolio, it becomes evident that the bulk is wind energy-based. However, there are some backlogs to full manifestation of this technology ranging from initial high cost to performance and reliability issues, among others. But in spite of these bottlenecks, new research trends have been assertive in seeking out a sustainable solution for harnessing wind energy for power generation especially in the design and construction of wind generators. In order to motivate and prime a sustainable energy mix among stakeholders, this paper is a shot at appraising the theory of these innovative wind generators towards ecological sustainability, economy, efficiency, and employment creation.
\end{abstract}

\section{Introduction}

Renewable energy is that source of energy which is abundant and continuously being replenished. No doubt, renewable energy comes with other benefits such as environmental friendliness, as well as being a source of electricity. The traditional means of generating electricity is by electrical generators running on fossil fuels e.g. petroleum and coal. The burning of fossil fuels to generate electricity is a dirty process, which leads to greenhouse gas (GHG) emissions vis-à-vis climate changes (Akuru et al, 2013).

While there are many sources of renewable energy, this paper will focus on wind energy, and it is too early to underscore this choice. In the interim, wind energy has been identified as the world's fastest growing source of renewable energy, grow- ing at a worldwide average of $30 \%$ annually (Swift and Chapman, 2007), Figure 1 shows this growth. The global wind report for 2013 indicates that wind energy markets trends soared more in 2013 than ever before, and is anticipated to get even better in the coming years (Global Wind Report, 2014).

The bottlenecks associated with the implementation of wind energy for power generation is as much as its advantages and they continue to evolve with time. There are issues bothering largely on the unpredictable nature of wind speed and direction, grid integration, and trapping of excess wind power for storage. Others are site location problems arising from noise, and habitat nuisance. The issue of transmission is also worrisome because wind power sites are usually remote from urban locations where the utility services are. Most of these problems lead overall to high initial capital costs, which have contributed to a growing quest for improvement in wind generator designs, as well as other components comprising wind energy conversion (WEC). Consequently, the focus here will be on the new generators that are being developed in order to make wind power generation more efficient, cheaper, and cleaner.

The process of converting wind energy to electricity is rather involved, moving through various interconnected stages. The next section is dedicated to offer a basic description on the operational background of the wind generator technology. Following after, Section 3 will review the conventional wind generator concepts which will provide a background for further deliberating on the newer models in Section 4. The paper finishes with some concluding remarks in Section 5.

\section{Overview of wind generator technology}

A good historical account on how the WEC technology started can be obtained from Ackermann and Söder (2005).Basically, the conversion is per- 


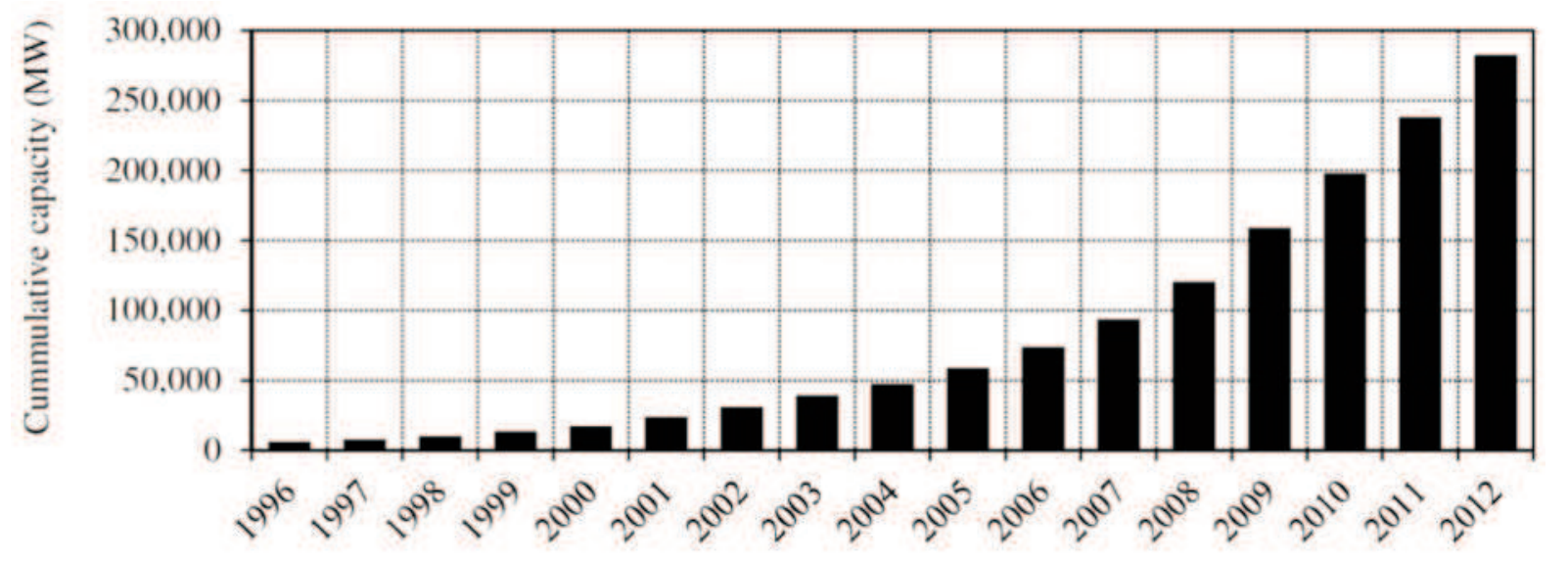

Figure 1: Wind energy: growth of worldwide cumulative installed total (Potgieter, 2014)

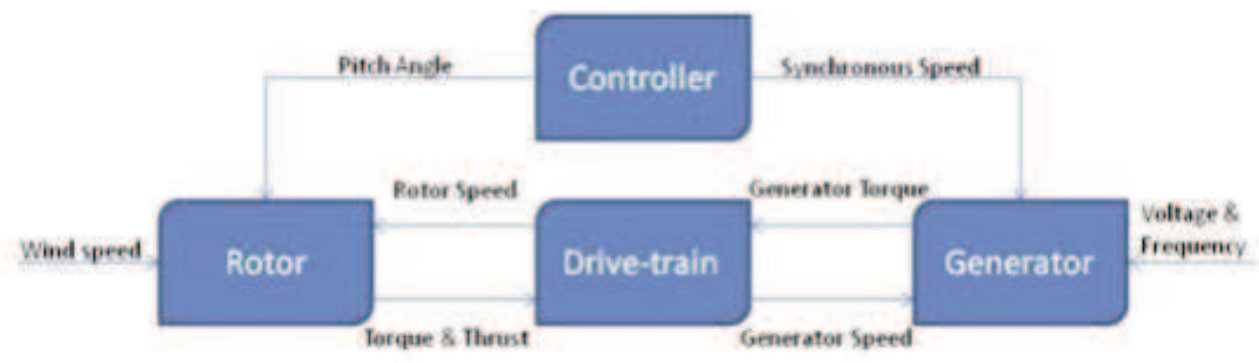

Figure 2: Process flowchart of a typical WEC system

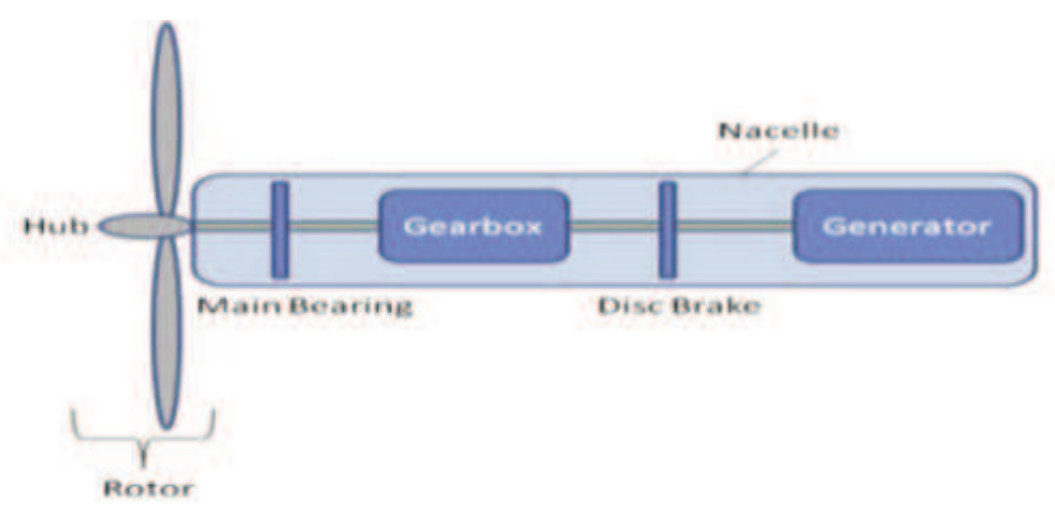

Figure 3: The main parts of a wind turbine

formed according to the flowchart as shown in Figured 2. Normally, wind energy is incident on a rotor, which then uses the mechanical energy produced to drive a generator that produces electricity, though the interconnection and control systems are also necessary components (Chang, 2002). This shows that the wind generator only forms a part of a complex WEC system called the wind turbine (Figure 3).

There are two basic configurations of wind turbines based on their axis of spin - the vertical axis (VAWT) and horizontal axis (HAWT) wind turbines - with the HAWT being the dominant model. The HAWT consist of a nacelle mounted on a tower. The nacelle is the part which contains the generator, the gearbox and the rotor. The gearbox provides options for altering the speed of the rotor to match the generator requirements. However, direct drive structures exist where newer generator designs are directly coupled to the rotors of wind turbines. The presence of gearboxes is usually responsible for the audible noise in wind turbines.

A wind turbine can also operate based on constant (fixed) or variable speed profiles, with variable speed offering greater efficiency. This is responsible for the system to be either geared or direct-drive generator types. Constant speed means that the wind turbine rotor speed is fixed irrespective of the wind speed, and it is determined by the grid frequency. The disadvantage of the constant speed system is its limited speed range leading to lower efficiency during wind fluctuations.

On the other hand, the power quality in variable speed systems is much better when compared with 
the constant speed systems due to a wider speed range, increased energy capture and reduced acoustic noise during wind fluctuations. To achieve variable speed mode, power electronic converters are used to provide constant frequency and constant voltage to the grid, whereas the rotor can independently vary. The disadvantage of the variable speed profile is more complex control and higher costs. Besides, wind turbines can be grouped into small, intermediate and large systems based on their output power capacity as shown in Table 1.

Wind speed is critical to the performance of a wind turbine; the stronger the wind the more power that will be available. As a matter of fact, doubling wind speed results in eight times more power considering the wind turbine output power expression in Abad et al. (2011) recalled in (1); where, $P_{t}$ is power recovered from wind energy, $\rho$ is air density, $R$ is radius of wind turbine, $V_{v}$ is wind speed and $C_{p}$ is the power coefficient. Other factors that determine the power output include height of the turbine and air temperature.

\section{$P_{t}=1 / 2 \rho \pi R^{2} V_{v}^{3} C_{p} \quad$ (1)}

In essence, wind generator technology is defined by four stages (Munteanu et al., 2008):

- The turbine rotor which interacts with the incoming wind;

- The drive trains which either transforms or couples the speed of the rotor to match with that of the generator;

- The generator that converts mechanical energy which it receives into electrical power; and

- The electronic and electrical subsystem for control and grid interconnection.

Table 1: Power rating of wind turbine systems

\begin{tabular}{lcc}
\hline Wind turbine & Power rating & Typical load coverage \\
\hline Small & Less than $100 \mathrm{~kW}$ & $\begin{array}{c}\text { Single homes } \\
\text { (Off-grid) }\end{array}$ \\
\hline Intermediate & $10-250 \mathrm{~kW}$ & $\begin{array}{c}\text { Villages or cluster } \\
\text { of homes (Off-grid) }\end{array}$ \\
\hline Large & Several MWs & $\begin{array}{c}\text { Wind farms } \\
\text { (Grid-connected) }\end{array}$ \\
\hline
\end{tabular}

\section{Conventional wind generator models}

The generators for wind turbines come in various structures, speed capabilities and sizes. Synchronous generators (both PM and non-PM types), as well as squirrel cage (SCIG) and woundrotor (WRIG) induction generators have been used for small, medium and high power wind turbines. However, DC generators are not popular because of high maintenance requirements due to brushes and need for DC-AC inverters.

\subsection{SCIG}

SCIG are typically applicable to constant-speed systems with direct grid-connection, but with some dis- advantages during gusty winds. In steady-state, the machine operates in over-synchronous regime synchronous speed exceeded by grid frequencyimposed rotational speed. The set-up of SCIG is shown in Figure 4a, and a summary of its advantages and disadvantages is shown in Table 2. To overcome the power factor issues, SCIG are equipped with capacitor banks for reactive power compensation. Also, when full converters are implemented, there is complete control of the active and reactive power exchanged with the grid (Abad et al., 2011). The soft-starter act to regulate the inrush currents during grid connection.

\subsection{WRIG}

A quick fix to SCIG is the WRIG (Figure 4b), which is incorporated with a variable external rotor resistor which can be adjusted to control the slip $(0-10 \%)$ of the generator, making it a basic variable-speed WEC system. This generator does not require slip rings because the additional variable rotor resistance can be changed using an optically controlled converter mounted on the rotor shaft. The control of the slip enables the power output in the system to also be controlled, and the fluctuations can be reduced.

\subsection{DFIG}

The doubly-fed induction generator (DFIG) is in a class of fully developed variable-speed generators, even though it is usually an improved WRIG version. It is acclaimed to be the leading technology in wind energy generation in recent years (Abad et al., 2011). As shown in Figure 4c, the doubly-fed connotation is as a result of this variety of WRIG being connected directly to the constant-frequency grid on the stator, while the rotor is fed by a back-toback (AC-AC) converter that is also connected to the grid. The stator outputs power into the grid, while the twin converters control the generator active and reactive power on the rotor side and DClink voltage on the grid side, respectively. It is mentioned that the size of the converters is not rated to the total generator power but to the selected speed variation range (Ackermann and. Söder, 2005).

When the slip is negative, the rotor feeds power into the grid and it absorbs power from the grid when the slip is positive, demonstrating that power flow is proportional to the slip. The main characteristics of DFIG have been summarised as follows (Abad et al., 2011):

- Limited but wider operating speed range $( \pm 30 \%)$;

- Small-scale power electronic converter (reduced power losses and price);

- Fully decoupled active and reactive power control;

- High maintenance due to slip-rings;

- Presence of gears. 
Table 2: Basic features of SCIG

\begin{tabular}{ll}
\hline \multicolumn{1}{c}{ Advantages } & \multicolumn{1}{c}{ Disadvantages } \\
\hline Mechanically simple & $\begin{array}{l}\text { Low full load power factor due to active and reactive } \\
\text { power compromises }\end{array}$ \\
\hline Low maintenance cost due to absence of brushes cost & Electrical fluctuations as a result of mechanical stress \\
\hline Very robust & Limited control \\
\hline Very stable & Need for gears \\
\hline
\end{tabular}

Higher efficiency is possible with two winding sets for

low and high wind speed (variable) operations or

combined effect of two SCIG

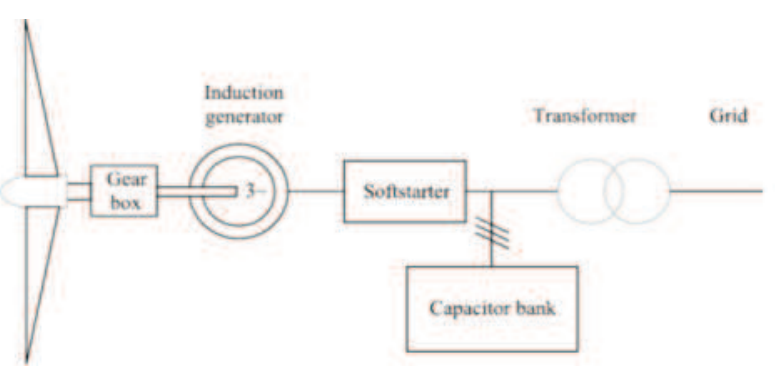

(a) SCIG

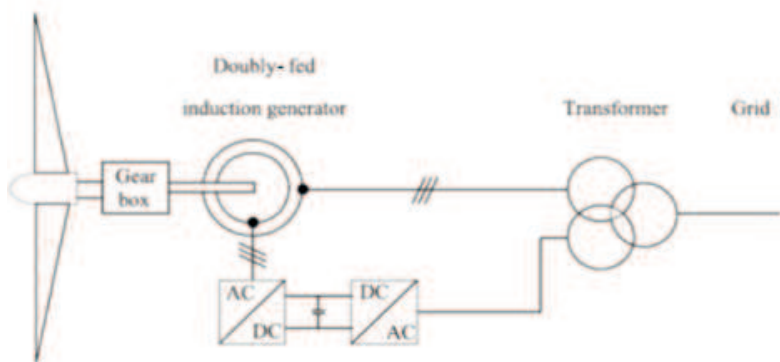

(c) DFIG

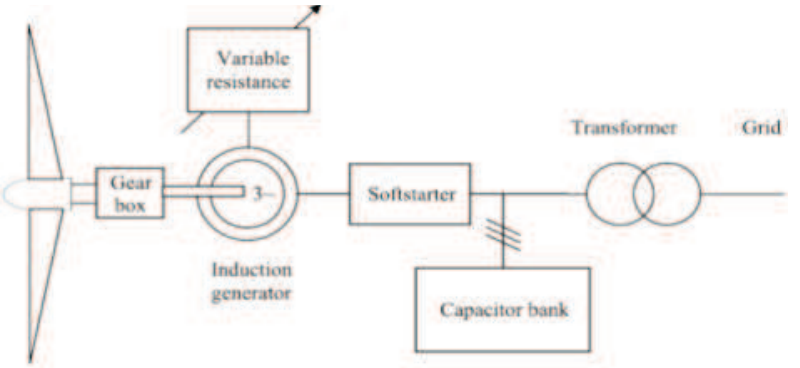

(b) WRIG

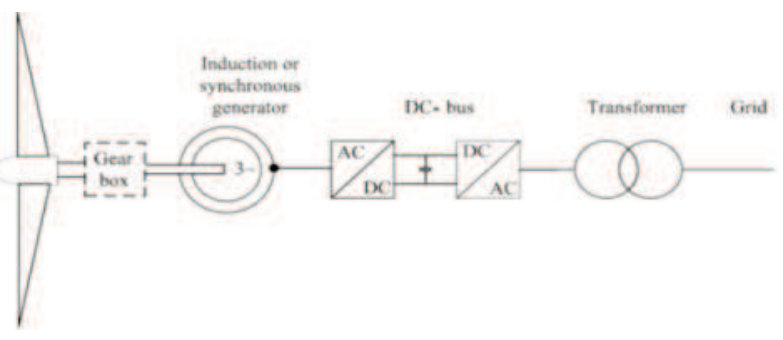

(d) VSWT

Figure 4a-d: Topologies of conventional wind generators (Munteanu et al., 2008)

\subsection{Synchronous generators}

Synchronous generators which is the brainchild of modern wind generators, can either be woundrotor (WRSG) or permanent magnet (PMSG), with the latter gaining more popularity due to key features such as considerable reduction in volume of excitation systems, higher air gap magnetic flux densities, better dynamic performance, and nowadays cheaper options (Gieras, 2008). They operate similar to DFIG with a back-to-back converter (rated to the generator power), but with higher efficiency. The grid-side converter provides voltage regulation, and the rotor of the wind turbine is not at a risk of losing synchronism because it is isolated from the grid. It can be operated as fixed speed or variable speed systems, offering advantages such as one-way speed control, wider speed range and longer air gaps which helps to improve stability (Madani, 2011).

The advantage of WRSG over PMSG is that it can by itself produce reactive power which it uses to regulate better voltage; however, because of the need of brushes it is usually limited among such wind generator options. Moreover, unlike PMSG, weight and size of WRSG increases sharply as pole numbers increase.

Salient pole PMSG operate at low speeds because of its higher pole numbers, and thus may not be geared for low and medium speed applications as pictured in Figure 4d. In WEC systems, a set-up without a gearbox offers huge advantages in that it removes the unconventional transformation from very low speeds to higher speeds. Synchronization during start-up, voltage and reactive power regulation and demagnetization due to high temperature exposures are some concerns in PMSG applications that are attracting renewed research. Overall, the many advantages of SG make them superior to IG as wind generators, its significant weight reduction being fundamental.

\section{Design trends in contemporary wind generators}

In the last three decades, progress in wind electric machine technology has seen newer topologies developed. Ragheb (2010) listed five major developments that describe modern wind machines as:

- Design of hub structures; 


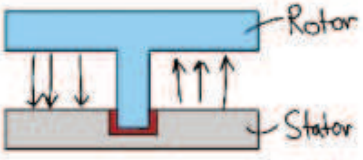

Axial-flux

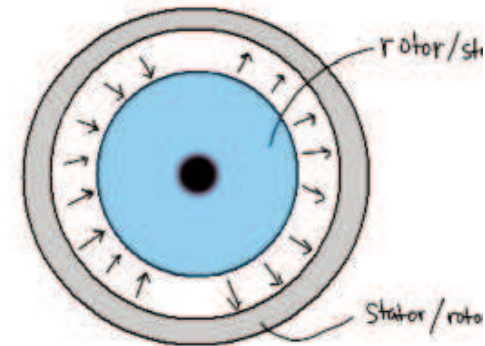

Radial. flux
Figure 5: Cross section of axial and radial flux direction in a typical PMSG, respectively

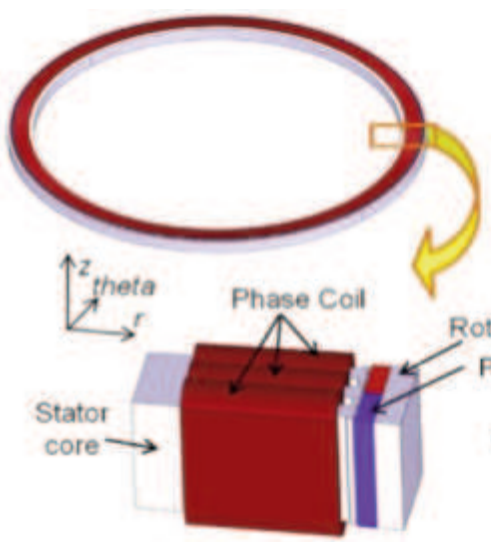

(a)

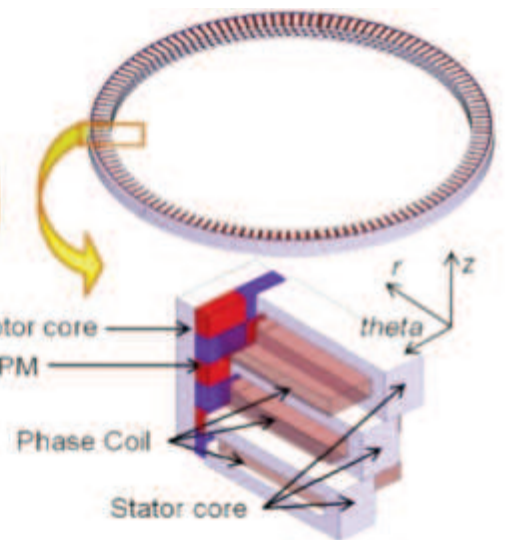

(b)

\section{Figure 6: Cut-out of (a) LFM, (b) TFM}

- Increase in higher capacity turbines reaching 5$10 \mathrm{MW}$;

- Preference for variable speed turbines;

- Adoption of gearless wind turbines;

- Evolution of permanent magnet excitation.

In another instance, Gieras (2008) identified some concepts that drive the technology advancement of electric machines today to include mechatronics, microelectromechanical systems (MEM), superconductivity (SC), solid state converters, energy conservation, power quality and sustainability. Gradually, there is a shift from the conventional wind generators which we have known to very unusual designs that offer superconducting excitation systems, efficient control, excellent power quality, weight and size reduction, environmental friendliness, improved performance, increased reliability, and even cost effectiveness. Some of these generator concepts are further discussed.

\subsection{PMSG}

In Madani (2011), the choice of PMSG for his wind generator design is predicated on the fact that they are more amenable to higher torque densities when compared to other contemporary machines for the same weight and size. However, since cost remains a major concern with PMSG, various topologies are being innovated for low speed and variable speed applications.

\subsubsection{Air gap orientation}

Axial flux (with disc type rotors) and radial flux (with cylindrical rotors) are two ways in which air gap orientation can be defined in PMSG as shown in Figure 5. If the flux direction is perpendicular to the axis of the air gap, the machine is called radial; whereas, if it is parallel with the air gap axis, it is axial. The conventional types of PMSG used in wind systems are radial machines (RFPM), and because of its popularity, cost of production is lower. Axial flux (AFPM) topologies have been the earliest electrical machines but were shelved for RFPM due to the following reasons (Gieras et al., 2008):

- Strong axial (normal) magnetic attraction force between stator and rotor;

- Fabrication difficulties in making slots;

- High cost of manufacturing;

- Difficulty in assemblage and air gap construction.

In recent times, breakthroughs in PM materials have revived the interest in axial flux machines, which possess high torque density and compactness, and are good candidates for low-speed directdrive wind systems since a large number of poles can be accommodated. However, a major drawback of AFPM is its very complicated manufacturing process.

\subsubsection{Direction of magnetic field loop}

Based on the flux path, electric machines can be categorized as longitudinal flux machines (LFM) or transverse field machines (TFM) - (see Figure 6). LFM has their flux path longitudinal or axial to the direction of motion, while TFM flux lines lie in planes transverse to direction of motion and their windings are torus or ring shaped. LFM are the conventional machines, having concentrated or distributed windings, and are good for direct drive applications because of their high torque and low torque ripple characteristics. TFM also possess high torque densities, but they possess torque ripples. The potentials of TFM in the wind system are manifest in their capacity to produce more power densities than conventional machines and have been suggested for large direct-drive PM wind generators due to its active mass reduction (Bang, 2010).

\subsubsection{Rotor positioning}

There are two possibilities on the position of rotors in wind generators - internal and external rotors (Figure 7). In external rotors, the rotor surrounds the stator outer diameter and usually houses the magnets in their inner circumference. The advantages of this structure are that the rotor is equipped with a greater pole number and can provide good 
support for the magnets. Outer rotors have been used in small HAWT. On the other hand, inner rotor machines are more common in wind generator applications, where they offer better cooling for the armature windings placed on the stator.

\subsubsection{Magnet arrangement}

PMSG can also be categorized based on the magnet arrangement in the rotor. The commonest types are the surface mounted permanent magnets (SMPM), where the magnet is mounted on the surface of the rotor. SMPM is the preferred configuration because of simple rotor geometry where there is no saliency, but there is high risk of demagnetization. Others are, inset and buried magnets. In inset machines, flux leakage is high because there are iron interpoles which create saliency. But in comparison with SMPM, they possess higher torque density due to additional reluctance torque making them good candidates for direct-drive applications in wind generators.

Inset machines are also called interior permanent magnet (IPM) machines. A major advantage of this structure is that weak PM materal such as ferrite can be employed. It is also good in preventing demagnetization. This topology is applicable in high-speed wind systems due to mechanical strength of the rotor against centrifugal force, but it is fraught with high flux leakage.

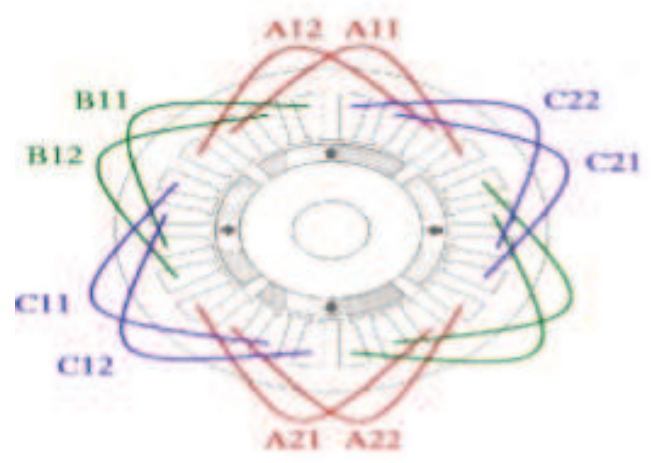

(a) 24-slot, over lapping (distrituted)

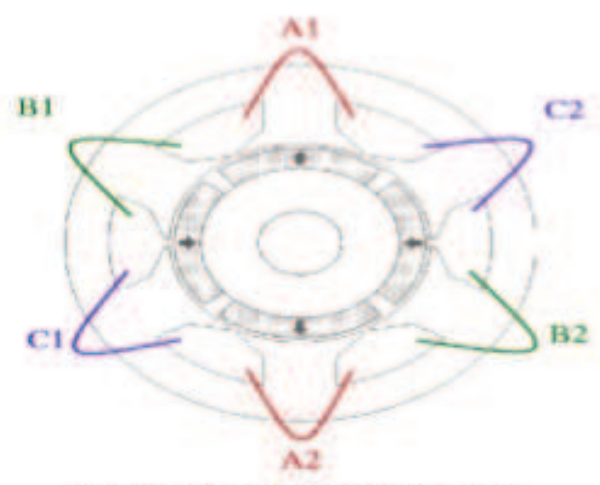

(e) 6-slot, non-overlapping, all teeth wound

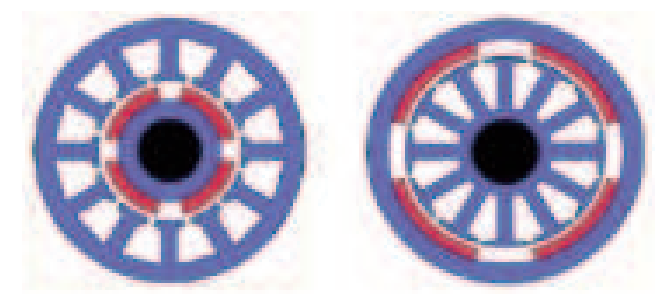

Figure 7: Internal (left) and external (right) rotor PMSG

\subsubsection{Winding selection}

Two types of windings are overlap and non-overlap windings. Overlap windings are the conventional windings which can either be distributed or concentrated. Distributed winding is associated with long end windings that result in higher copper losses and longer axial lengths, a key disadvantage. Contrary, concentrated windings are built with shorter end windings that lead to reduced joule losses, high efficiency and fault-tolerant applications in PMSG for wind systems.

Concentrated winding is a terminology which is becoming familiar with non-overlap windings. Nonoverlap (fractional-slot) windings bring an improvement to traditional concentrated windings in that it is possible to have reduced mutual coupling among phases, reduced manufacturing costs, low torque ripple, as well as higher winding factors and higher

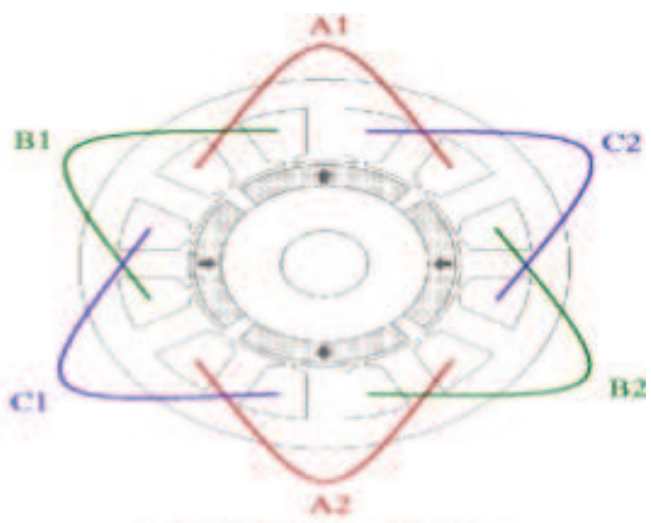

(b) 12-sles, overtapping (comeentrated)

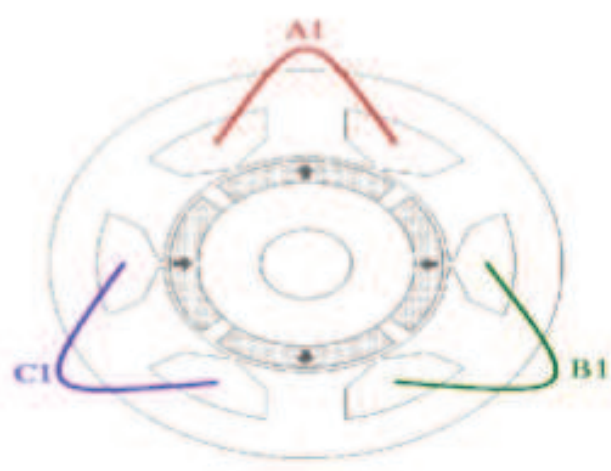

(d) 6-slot, non-overlapping. alternate teeth wound

Figure 8: Typical stator winding configurations 
torque densities. Multiple layers are possible as this reduces the harmonic content of the MMF which is a major drawback. It is reckoned that these MMF harmonics cause an alternating magnetic field in the rotor giving rise to rotor losses (Libertt and Souland, 2003). It has also been reported that employing concentrated windings in PM generators for low speed direct-drive applications is very attractive because of their simple structure, higher pole numbers and shorter-end windings (Gieras et al., 2008). Figure 8 shows the various winding configurations discussed.

\subsection{Drivetrains}

Two drivetrains have been mentioned earlier: geared and direct-drive (gearless) systems. Until recently, wind generation systems consisted of a gearbox connected to high-speed variable speed generators. Today, wind energy systems can be distinguished by the presence or absence of gears. Gearless systems are still very new, and are largely due to advances in PM generators.

\subsubsection{Geared}

The function of a gearbox is to transform mechanical speed from low to high speed by means of a step-up system. Two main configurations in use are the single-stage (1G) and three-stage (3G) geared drivetrains. In the $1 \mathrm{G}$ concept, the generator, gearbox, main shaft, and shaft bearing are all integrated within a common housing. Multiple-gearbox schemes like the $3 \mathrm{G}$ concept is designed to further reduce the generator size and improve efficiency in variable wind concepts, and is usually connected to a full-scale power converter leading to losses more than in the other drive concepts. Moreover, it is costly, decreases drive efficiency and needs maintenance. The main advantage of geared systems is their ability to ensure a wider speed range.

\subsubsection{Direct-drive}

This concept requires that $S G$ is directly connected to the turbine without making use of gears. Actually, WRSG and PMSG are applicable to this technolo$\mathrm{gy}$, but the future is more with PMSG. The advantages of this scheme are simple drivetrains, high efficiency, reliability, low speed applications and absence of noisy gear boxes, thus making it a cheaper bargain. There are also hybrid drivetrain systems that utilize a medium-speed generator, which combines fewer gearing stages and converter-fed PMSG.

\subsubsection{Full converter concepts}

There are three converter concepts based on the speed drivetrain of a wind generator, namely; highspeed (HSFC), medium-speed (MSFC) and lowspeed (LSFC) full converter concepts. Their characteristics are summarised in Table 3.
Table 3: Electrical drivetrain concepts

\begin{tabular}{|c|c|c|c|}
\hline Parame & & MSFC & LSFC \\
\hline Speed $r$ & ange & $2000 \mathrm{rpm}$ & $500 \mathrm{rpm} 30 \mathrm{rpm}$ \\
\hline Weight & Lowest & Intermediate & Highest \\
\hline Size & Smallest & Intermediate & Largest \\
\hline \multicolumn{3}{|c|}{ Gearbox Present (3G) } & Present $(2 G / 1 G)$ Absent \\
\hline \multicolumn{2}{|c|}{$\begin{array}{l}\text { Generator } \\
\text { Synchronous }\end{array}$} & \multicolumn{2}{|c|}{$\begin{array}{l}\text { Asynchronous/Synchronous } \\
\text { Synchronous }\end{array}$} \\
\hline
\end{tabular}

\subsection{Power electronics}

Another concept that has impacted the development of new wind generators is the strides recorded in power electronics technology. Power electronics have developed various topologies of converters such as back-to-back, matrix and multi-level converters. Some of the achievements of power electronics by means of efficient power converters are in gearless variable speed control, power amplification, power conversion, grid interconnection, and frequency/voltage regulation. Although, a major setback is that they contribute to overall system losses and cost implications.

\subsection{Innovative wind generator concepts}

Before now, the dominating wind generators have been DC commutating machines, AC induction and AC synchronous machines. Presently, there are far too many other concepts for wind generating systems that cannot be fully debated in this paper because they are either both developed but are yet established for wind generating systems, or are still relatively new and are being tested, or have been proposed and information about them is very scanty. However, a number of them will be mentioned here for reference purposes:

- Synchronous reluctance generator (SynRG) (Tokunaga et al; 2011)

- Switched reluctance generator (SRG) (Choi et al, 2014);

- Brushless DFIG (BDFIG) (Long et al., 2013);

- Split-PM induction generator (S-PMIG) (Potgieter, 2011);

- Slip synchronous-PM generator (SS-PMG) (Potgieter, 2014);

- Switched-flux generator (SFG) (Sulaiman et al., 2013; Kayano et al., 2010; Ojeda et al., 2012)

- Ferrite-assisted reluctance synchronous generator (FA-RSG) (Baek et al, 2009);

- Magnetic gears and pseudo-direct drive (Wenlong, 2011);

- Superconducting machines (Jensen et al, 2013);

- PM Field-intensified synchronous reluctance generator (FI-RSG) (theoretical).

\subsection{Leading manufacturers and countries}

There have been a number of manufacturers leading the research, design and production of wind turbine concepts and generating systems worldwide. 
Key among them are Vestas (Denmark), GE (USA), Enercon (Germany), Gamsea (Spain), Suzlon (India), Siemens (Germany), Repower (Germany), Nordex (Germany), Ecotècnia (Spain), Mitsubishi (Japan), JSW (Japan), ScanWind (Norway), Vensys, STX Windpoer, EWT, MTorres, Leitwind, and Goldwin (Ragheh, 2010; Bang, 2010). Over the years, some of these manufacturers get bought over by larger companies; for instance GE bought ScanWind in 2009. Vestas Wind Systems A/S is the largest manufacturer in the world with a total 51547 wind turbines installed at a capacity of over 31188 MW in 73 countries (Vestas, 2014).

The world leader in wind power installed capacity is China with over 90 GW installed capacity, while Germany remains Europe's superpower at 34.25 GW (Global Wind Report, 2014). Egypt has the largest capacity in Africa at $550 \mathrm{MW}$, followed by Morocco and Ethiopia. The recent annual growth in wind power installations have been put at 12 per cent, and certainly this trend will continue to intensify.

\section{Conclusion}

Wind generators are integral to wind energy conversion systems as revealed by the review undertaken in this paper. Little wonder, the vista of electrical machines in recent years, where wind energy has received growing attention, has surpassed any era of its existence. This is evident in the fact that all aspects of the traditional electrical machine is being dissected and routinely transformed to meet growing demands of this time. There are many considerations for this development as already highlighted in this paper that have specially considered one category of electrical machines - wind generators. The advantages of wind power generation, in spite of obvious challenges, are huge. Wind power generation leads to cleaner power options, as well as a source of income. There are many other benefits like the impact of computational modelling and simulation that are critical to contemporary designs of wind generators, but have not been expounded here. Therefore, the emphasis of this revision is an immediate call to action for sustained renewable energy deployment as an immediate alternative to fossil fuels, bearing in mind that wind is abundant, more amenable to large power capture, safe, and somehow, cheap.

Also worthy of mention, is the fact that electricity generation have always been electrical generatorbased and so, wind energy generation is closest to conventional electricity generation, as what is only required is to change the source of fuel in these machines into wind. The potential of using modern wind generators in sites where wind supply is very low has been demonstrated (Kayano et al., 2010), making it attractive for all and sundry. Then of course, there is the increasing drive for research and development in wind power generation and the promise it holds for a sustainable energy mix. Last of all, one remarkable revelation in this evaluation is the bias for direct-drive applications in the design of modern wind generators.

\section{References}

Ackermann, T. and Söder, L. (2005). Wind Power in Power Systems: An Introduction, in Wind Power in Power Systems (ed T. Ackermann), Chichester, UK: John Wiley \& Sons.

Abad, G., López, J., Rodríguez, M., Morroyo, L. and Iwanski, G. (2011). Doubly Fed Induction Machine: Modeling and Control for Wind Energy Conversion Hoboken, New Jersey: John Wiley \& Sons.

Akuru, U.B., Okoro, O.I. and Chikuni, E: Impact of Renewable Energy Deployment on Climate Change in Nigeria, AFRICON, 2013, 9-12 Sept. 2013 doi: 10.1109/AFRICON.2013.6757649.

Baek, J., Rahimian, M.M. and Toliyat, H.A.: (2009).Optimal design of PM assisted synchronous reluctance generators using lumped parameter model and Differential Evolution Strategy, Energy Conversion Congress and Exposition, 2009. ECCE 2009. IEEE, Vol., No., pp.2453, 2459, 20-24 Sept. 2009 doi: 10.1109/ECCE.2009.5316225.

Bang, D. (2010). Design of a transverse flux permanent magnet machines for large direct-drive wind turbines. MEng thesis, Pukyong National University, Korea.

Chang, L. (2002). Wind Energy Conversion Systems, IEEE Canadian Reviews-Spring, pp.12-16.

Choi, D-W., Byun, S-I. and Cho, Y-H. (2014). A Study on the Maximum Power Control Method of Switched Reluctance Generator for Wind Turbine, Magnetics, IEEE Transactions on, vol.50, no.1, pp.1,4, Jan. 2014 doi: 10.1109/TMAG.2013.2274174.

Gieras, J.F. (2008) Advancements in Electric Machines, Milton Keynes, United Kingdom: Springer.

Gieras, J.F., Wang, R-J. and Kamper, M.J. (2008). Axial Flux Permanent Brushless Machines, Second edition: Springer.

Global Wind Report: Annual Market Update 2013. Global Wind Energy Council (GWEC), April 2014.

Jensen, B.B., Mijatovic, N. and Abrahamsen, A.B. (2013). Development of superconducting wind turbine generators J. Renew. Sustain. Energy, Vol. 5, No. 2, pp. 023137-1-02313712.

Kayano, S., Sanada, M. and Morimoto, S. (2010). Power characteristics of a permanent magnet flux switching generator for a low-speed wind turbine, Power Electronics Conference (IPEC), 2010 International , vol., no., pp.258,263, 21-24 June 2010 doi: 10.1109/IPEC.2010.5543884.

Libert F. and Soulard, J. (2003). Design Study of a Direct-Driven Surface Mounted Permanent Magnet Motor for Low Speed Application, Symposium on Advanced Electromechanical Motion Systems Electromotion 2003, Vol. 1 pp. 252-257, 26-28 November 2003, Marrakesh, Morocco. 
Long T., Shao S., Abdi E., McMahon R.A. and Liu, S. (2013). Asymmetrical Low-Voltage Ride Through of Brushless Doubly Fed Induction Generators for the Wind Power Generation, Energy Conversion, IEEE Transactions on, Vol. 28, No. 3, pp.502,511, Sept. 2013doi: 10.1109/TEC.2013.2261818

Madani, N. (2011). Design of a permanent magnet synchronous generator for a vertical axis wind turbine. M Sc thesis, School of Electrical Engineering, Royal Institute of Technology, Stockholm, Sweden.

Munteanu, I., Bratcu, A.I., Cutululis N-A. and Ceanga, E. (2008). Optimal Control of Wind Energy Systems towards a Global Approach, Springer.

Ojeda, J., Simoes, M.G., Guangjin L. and Gabsi, M. (2012). Design of a Flux-Switching Electrical Generator for Wind Turbine Systems, Industry Applications, IEEE Transactions on, vol.48, no.6, pp.1808, 1816, Nov.-Dec. 2012 doi: 10.1109/TIA.2012.2221674.

Potgieter, J.H.J. (2011). Design and analysis of a gearless direct grid permanent magnet induction generator. Masters thesis, University of Stellenbosch, Stellenbosch, South Africa.

Potgieter, J.H.J. (2014). Optimal topology and critical evaluation of slip synchronous permanent magnet wind generator. $\mathrm{PhD}$ thesis, University of Stellenbosch, Stellenbosch, South Africa,

Ragheb, M. Modern Wind Generators, NetFiles. University of Illinois at Urbana-Champaign, 28 Feb. 2010. Web 2 June 2014.

Sulaiman, E., and Teridi, M.F.M., Husin, Z.A., Ahmad, M.Z. and Kosaka, T. (2013. Investigation on Flux Characteristics of Field Excitation Flux Switching Machine with Single FEC Polarity, Procedia Technology, Volume 11, Pages 572-579, ISSN 2212 0173, http://dx.doi.org/10.1016/j.protcy.2013.12.230.

Swift A. and Chapman, J. A Primer on Wind Energy: Meteorology, Technology, Economics and Transmission, presented at the University of Texas School of Law 2007 Wind Energy Institute Conference, Austin, Texas, February 2007, p. 1.

Tokunaga, S. and Kesamaru, K. (2011). FEM simulation of novel small wind turbine generation system with synchronous reluctance generator, Electrical Machines and Systems (ICEMS), 2011 International Conference on , pp.1,6, 20-23 Aug. 2011 doi: 10.1109/ICEMS.2011.6073968.

Vestas track record as per $31 \mathrm{March}, 2014$. Available at: http://www.vestas.com/en/about/track_record\#!.

Wenlong, L., Chau, K.T. and Jiang, J.Z.: Application of Linear Magnetic Gears for Pseudo-Direct-Drive Oceanic Wave Energy Harvesting, Magnetics, IEEE Transactions on, Vol. 47, No.10, pp.2624,2627, Oct. 2011 doi: 10.1109/TMAG.2011.2146233. 